\title{
Dynamics of an inverted pendulum with two concentrated masses
}

\author{
Bin Zhen ${ }^{1, a}$, Zhenyu Fu ${ }^{2, b}$ and Liang Chang ${ }^{3, c}$ \\ ${ }^{1}$ School of Environment and Architecture, University of Shanghai for Science and Technology, \\ Shanghai 200093, P.R. China \\ 2 School of Civil Engineering and Architecture, Nanchang Hangkong University, \\ Nanchang, Jiangxi 330063, P.R. China \\ azhenbin80@163.com, blitaosfive@163.com, cchangliang@nchu.edu.cn
}

\begin{abstract}
Keywords: inverted pendulum; stability chart; perturbation method; Mathieu equation
Abstract. In this paper, we investigate the dynamics of an inverted pendulum with two concentrated masses. The perturbation method is used to find the transition curves. We emphasize the difference between the case of two concentrated masses and that of one concentrated mass. Numerical simulations are carried out to demonstrate the influence of the distribution of mass of the pendulum on the stability of the inverted pendulum. Our research shows that in some cases the inverted pendulum with two concentrated masses has to be considered to analyze the stability. It may be error if the inverted pendulum with one concentrated mass is used to find the transition curves and corresponding periodic solutions.
\end{abstract}

\section{Introduction}

Pendulum has attracted intense interest from scientists in physics, mechanics and material science over several centuries. As the earliest application, Galileo used the pendulum to measure the time based on the property of isochronism. The analysis of motion of a simple planar pendulum has received a lot of interest for many years because of potential applications. The pendulum subjected to periodic forcing may be used as a good approximate model of many physical systems, such as off-shore structure [1], the response of Josephson junctions, crane barges [2], and so on. A simple pendulum has two equilibrium configurations: the ball located in the down or the up position. The down position is stable, and the other unstable. If the pivot point is rapidly driven by a vertical periodic forcing or vibrating then the inverted position can be stable for a range of forcing or vibrating amplitudes and frequencies. In this case, the pendulum contains some parameters that can vary as functions of time independently of state variables. The "dynamic stabilization" phenomenon of the inverted position, which has been noticed for a very long time, was observed experimentally [4,5]. Periodic oscillations, rotations even chaos [3] can occur in the simple pendulum model. The dynamic stabilization of the inverted pendulum has been demonstrated to play a role in the studies of the theory of accelerators, magneto plasma [6] as well as the walking motion of a pedestrian [7,8,9].The influence of mass distribution and length distribution of the inverted pendulum is still unknown for the walking motion of a pedestrian.

It should be noted that almost all inverted pendulum models were investigated by considering that all masses are concentrated at one end. However, it may be not appropriate for some cases, such as using the inverted pendulum to model a pedestrian's walking motions. In this paper we attend to analyze the dynamics of an inverted pendulum with two concentrated masses. We will use the perturbation method to analyze and find the transition curves. The difference between two cases that two concentrated masses and one concentrated mass for an inverted pendulum is demonstrated theoretical and numerically.

The rest of the paper is organized as follows: the inverted pendulum mode with two concentrated masses is introduced in next section. Then the perturbation method is used to find the transition curves of the inverted pendulum in a new section. Comparison and analysis are given to demonstrate 
the influence of distribution of mass of the inverted pendulum on its stability in the penultimate section. Conclusions are drawn in the last section.

\section{The inverted pendulum with two concentrated masses and its governing equation}

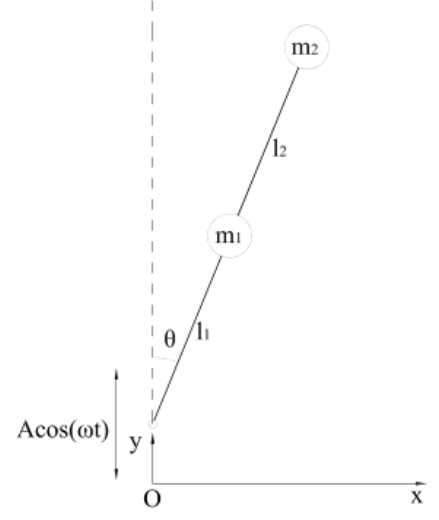

Fig.1 Schematic of the inverted pendulum with two concentrated masses whose pivot point undergoes vertical oscillations in the coordinate system

Consider the inverted pendulum shown in Fig.1, which consists of two masses $m_{1}$ and $m_{2}$ fixed at the distances $l_{1}$ and $l_{2}$ from pivot point, respectively. We assume that the pivot is subjected to a vertical oscillation $y=A \cos (\omega t)$, in which $\omega$ is the angular-driving frequency, $A$ is the amplitude of the oscillation, and $t$ is the time. We denote the rotation angle of the inverted pendulum around the pivot by $\theta$, the positive direction of which measures clockwise from the up position. The coordinates of pivot point and two concentrated masses are separately given by

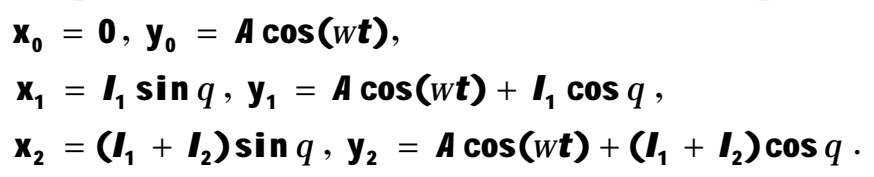

Then the kinetic and potential energies of the inverted pendulum can be written as

$$
\begin{aligned}
& T=\frac{1}{2} m_{1}\left(x \psi^{2}+y \psi^{2}\right)+\frac{1}{2} m_{2}\left(x \psi^{2}+y \psi^{2}\right), \\
& V=m_{1} g y_{1}+m_{2} g y_{2} \text {. }
\end{aligned}
$$

Using the Lagrange's equation

$$
\frac{d}{d t}\left(\frac{\partial}{\partial \theta^{\alpha}}-\frac{\partial}{\partial \theta} T+V=0\right. \text {, }
$$

and considering $\theta$ is small, the governing equation of the inverted pendulum can be obtained

$$
\theta+(\delta+\varepsilon \cos t) \theta=0 \text {, }
$$

where

$$
\delta=-\frac{g}{\omega^{2}} \frac{m_{1} l_{1}+m_{2}\left(l_{1}+l_{2}\right)}{m_{1} l_{1}^{2}+m_{2}\left(l_{1}+l_{2}\right)^{2}}, \varepsilon=A \frac{m_{1} l_{1}+m_{2}\left(l_{1}+l_{2}\right)}{m_{1} l_{1}^{2}+m_{2}\left(l_{1}+l_{2}\right)^{2}} \text {. }
$$

Eq.(7) is a Mathieu equation. In the following, we discuss the stability of Eq.(7) and the influence of parameters $\delta$ and $\varepsilon$.

\section{Stability analysis}

In this section, we use the perturbation method to calculate the transition curves of Eq.(7) and then discuss the influence of parameters $\delta$ and $\varepsilon$ on them. We assume that $\delta$ and $\theta$ can be separately written in the form of an expansion series as

$$
\delta=\delta_{0}+\delta_{1} \varepsilon+\delta_{2} \varepsilon^{2}+\mathrm{L},
$$




$$
\theta=\theta_{0}+\theta_{1} \varepsilon+\theta_{2} \varepsilon^{2}+\mathrm{L},
$$

where $\delta_{1}$ are the constants to be determined. Substituting Eqs.(8) and (9) into Eq.(7) and equating the coefficients of each power of $\varepsilon$ to be zero, one has

$$
\begin{aligned}
& \delta_{0}+\delta_{0} \theta_{0}=0, \\
& \delta_{1}+\delta_{1} \theta_{1}=-\theta_{0}\left(\cos t+\delta_{1}\right), \\
& +\delta_{0} \theta_{2}=-\theta_{1}\left(\cos t+\delta_{1}\right)-\theta_{0} \delta_{2} .
\end{aligned}
$$

Solving Eqs.(10)-(12), we have the following transition curves: $\delta=-\frac{1}{2} \varepsilon^{2}$, which emanates from the origin; and $\delta=\frac{1}{4} \pm \frac{1}{2} \varepsilon-\frac{1}{8} \varepsilon^{2}$, which emanates from $\delta=\frac{1}{4}$. The results are depicted in Fig.2.

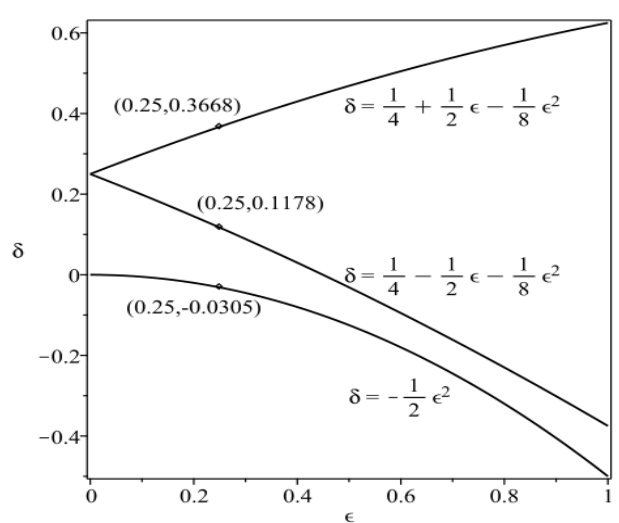

Fig.2 The transition curves of $\operatorname{Eq}(7)$

\section{Discussion of parameters}

In this section, we analyze how the parameters $m_{i}$ and $l_{i}$ affect the stability of Eq.(7). By letting $\mathrm{m}_{1}+\mathrm{m}_{2}=\mathrm{m}, \mathrm{l}_{1}+\mathrm{l}_{2}=\mathrm{l}$, we denote $\mathrm{M}=\frac{\mathrm{m}_{1}}{\mathrm{~m}}$ and $\mathrm{L}=\frac{\mathrm{l}_{1}}{\mathrm{l}}$. Then the parameters $\delta$ and $\varepsilon$ defined in Eq.(7) can be rewritten as

$$
\delta=-\frac{g}{\omega^{2}} \frac{\mathrm{ML} / 1+(1-\mathrm{M}) / 1}{\mathrm{ML}^{2}-\mathrm{M}+1}, \varepsilon=\mathrm{A} \frac{\mathrm{ML} / \mathrm{l}+(1-\mathrm{M}) / \mathrm{l}}{\mathrm{ML}^{2}-\mathrm{M}+1} \text {. }
$$

We first carry out numerical simulations of the transition curves by separately letting $\mathrm{L}=0.1,0.2$, 0.3, 0.4 and $\mathrm{M}=0.9$. The results are presented in Fig.3. Then we fix $\mathrm{L}=0.1$ and let $\mathrm{M}=0.9,0.8,0.6$ and 0.3 , respectively, results are shown in Fig.4.

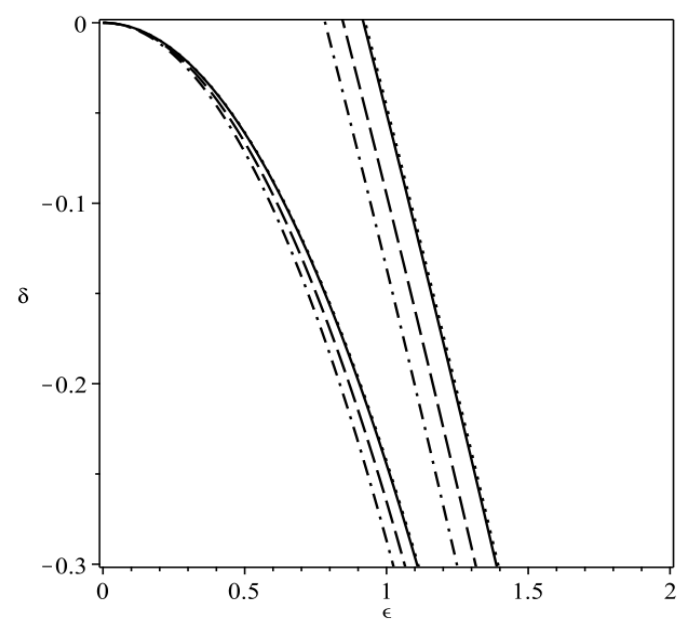

Fig. 3 The transition curves for $\mathrm{L}=0.1$ (dot dash line), $\mathrm{L}=0.2$ (dot line), $\mathrm{L}=0.3$ (full line) and $\mathrm{L}=0.4$ (imaginary line) while $\mathrm{M}=0.9$. 


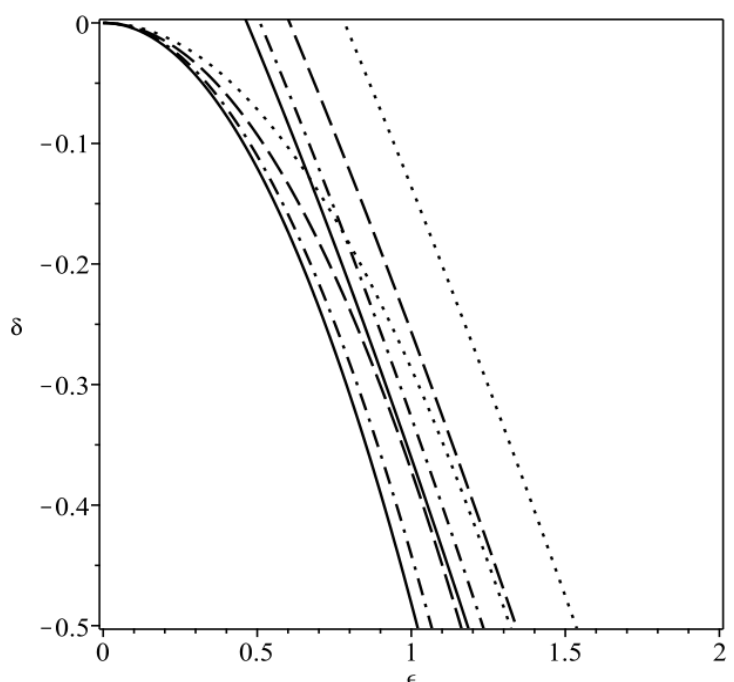

Fig. 4 The transition curves for $\mathrm{M}=0.9$ (dot line), $\mathrm{M}=0.8$ (imaginary line), $\mathrm{M}=0.6$ (dot dash line) and $\mathrm{M}=0.3$ (full line) while $\mathrm{L}=0.1$.

\section{Conclusions}

In this paper, we investigate the stability of an inverted pendulum with two concentrated masses. Different from other research, we discuss the influence of distribution of mass of the inverted pendulum on its stability. Figs. 4 and 5 show that When L is changing from 0 to 1 with a fixed M, the transition curve emanating from the origin starts to swing up till $\mathrm{L}=0.3$, and later with the increase of $\mathrm{L}$ this transition curve begins to swing down until it returns to the position at the very beginning. During this process, the other transition curve has the opposite variation and it's worth noting that the intersection of the curve and the $\delta$-axis is no longer a fixed value $1 / 4$ when $\mathrm{L}$ is not equal to 0 or 1. When $\mathrm{M}$ is increasing from 0 to 1 with a given $\mathrm{L}$, the two transition curves have the same performance of swinging up. Integrating the two transition curves in Figs.4 and 5, we can get the value of stability region area. Numerical comparison demonstrates that L has no effect on the size of stability zone area, while stability domain area will decrease.

\section{Acknowledgements}

This work was financially supported by the National Natural Science Foundation of China (Grant Nos.11472160 and 11672185), the financial aid from Natural Science Foundation of Jiangxi Province (20161BAB216103) and the project of Jiangxi Education Department (GJJ160708).

\section{References}

[1] A. Sofroniou and S. R. Bishop: submitted to Chaos Solitons \& Fractals (2006)

[2] F. J. McCormick, J. A. Witz: submitted to Journal of Underwater Technology (1993)

[3] S. R. Bishop, A. Sofroniou and P. Shi: submitted to Chaos Solitons \& Fractals (2005)

[4] H. P. Kalmus: submitted to American Journal of Physics (1970)

[5] H. J. T. Smith and J. A. Blackburn: submitted to American Journal of Physics (1992)

[6] M. M. Michaelis: submitted to American Journal of Physics (1985)

[7] W. K. Michael, B. Timothy and P. S. James: submitted to Gait \& Posture (2014)

[8] P. T. Cheng, C. M. Wang, C. Y. Chung and C. L. Chen: submitted Clinical Rehabilitation (2004) 
[9] C. Maurer and R. J. Peterka: submitted to Journal of Neurophysiology (2005) 